OPEN ACCESS

Edited by:

Eloisa Colin-Ramirez,

University of Alberta, Canada

Reviewed by:

Astrid Ruiz Margain,

Instituto Nacional de Ciencias

Médicas y Nutrición Salvador Zubirán

(INCMNSZ), Mexico

Elisa Gomez,

Universidad Anáhuac

Querétaro, Mexico

*Correspondence:

Qun Zhao

zhaoqun@hebmu.edu.cn

Specialty section

This article was submitted to

Clinical Nutrition

a section of the journal

Frontiers in Nutrition

Received: 18 July 2021 Accepted: 07 October 2021

Published: 28 October 2021

Citation:

Ding P, Guo H, Yang P, Sun C, Tian Y,

Liu Y, Li Y and Zhao Q (2021) Association Between the Nutritional Risk and the Survival Rate in Newly

Diagnosed GIST Patients. Front. Nutr. 8:743475.

doi: 10.3389/fnut.2021.743475

\section{Association Between the Nutritional Risk and the Survival Rate in Newly Diagnosed GIST Patients}

\author{
Ping'an Ding ${ }^{1}$, Honghai Guo ${ }^{1}$, Peigang Yang ${ }^{1}$, Chenyu Sun ${ }^{2}$, Yuan Tian ${ }^{1}$, Yang Liu ${ }^{1}$, \\ Yong $L^{1}{ }^{1}$ and Qun Zhao ${ }^{1 *}$ \\ ${ }^{1}$ The Third Department of Surgery, The Fourth Hospital of Hebei Medical University, Shijiazhuang, China, ${ }^{2}$ Internal Medicine, \\ AMITA Health Saint Joseph Hospital Chicago, Chicago, IL, United States
}

Background: Currently, the incidence of gastrointestinal stromal tumors (GIST) is increasing rapidly worldwide. Malnutrition may increase the risk of perioperative complications and affect the prognosis of patients. However, previous studies on the nutritional status of GIST patients and its impact on prognosis are limited. Therefore, this study aims to explore the incidence of malnutrition in newly diagnosed GIST patients, the proportion of participants in need of nutritional intervention, and the relationship between nutritional status and overall survival (OS).

Methods: We retrospectively analyzed the clinical data of GIST patients treated in our hospital from January 2014 to January 2018. Nutritional Risk Screening 2002 (NRS2002) and Patient-Generated Subjective Global Assessment (PG-SGA) were used to assess the nutritional status of all patients. This study was to investigate the clinical significance of PG-SGA by analyzing the relationship between PG-SGA score and OS.

Results: A total of 1,268 newly diagnosed GIST patients were included in this study, of which $77.76 \%$ were at risk of malnutrition (NRS2002 score $\geq 3$ ), and the incidence of malnutrition was $10.09 \%$ (PG-SGA score $\geq 4$ ). Meanwhile, we found $2.29 \%$ of the patients required urgent nutritional support (PG-SGA score $\geq 9$ ). Multivariate analysis showed that age $(p=0.013)$, BMl $(p=0.001)$, weight loss $(p=0.001)$, anemia $(p$ $=0.005)$, pre-albumin $(p=0.010)$, albumin $(p=0.002)$, tumor location $(p=0.001)$, tumor size $(p=0.002)$, and $\mathrm{NIH}$ classification $(p=0.001)$ were risk factors for nutritional status. The prognosis was significantly in GIST patients with different PG-SGA score at admission $(p<0.05)$.

Conclusion: This study suggested that malnutrition is common in newly diagnosed GIST patients, and the higher the PG-SGA score, the worse the clinical outcome.

Keywords: nutrition status, PG-SGA, NRS2002, gastrointestinal stromal tumors (GIST), risk factor, prognosis 


\section{INTRODUCTION}

Gastrointestinal stromal tumors (GIST) is the most common mesenchymal tumor in the gastrointestinal tract, which is often caused by the mutation of KIT and PDGFRA genes $(1,2)$. The incidence of GIST is increasing at an alarming rate worldwide (3). Numerous studies have demonstrated that nutritional deficiency account for about $85 \%$ of all patients diagnosed with cancer, of whom about $50-90 \%$ have weight loss and malnutrition at the beginning of treatment (4-6). Malnutrition in patients with malignant tumors can lead to low immune function, increase perioperative infection rates, prolong hospitalization time, increase medical costs, and more importantly, affect quality of life and prognosis (7-9). In addition, the relative risk of cancer deaths caused by malnutrition is 1.8 times that of patients without malnutrition $(10,11)$. Therefore, it is of great importance to assess the nutritional status of patients during cancer treatment to comprehensively evaluate their tolerance to treatment.

The incidence of malnutrition in gastrointestinal tumors, especially upper gastrointestinal tumors, is higher than that in non-gastrointestinal tumors $(12,13)$. However, most studies investigating the gastrointestinal tumors focus on the nutritional status of non-GIST cancers, such as gastric cancer and colon cancer, while few people pay attention to the nutritional status of GIST patients, and there is no consensus on nutritional guidelines for GIST patients. As the symptoms of GIST are usually non-specific, most patients are in advanced stage at the time of diagnosis. The oppression or obstruction caused by the tumor leads to malabsorption. This results in higher risk of malnutrition, especially in patients undergoing perioperative treatment and patients with advanced and recurrent metastasis $(14,15)$. Therefore, correction of the malnutrition in GIST patients is particularly important for improving the quality of life and significantly prolonging the survival period.

Currently, the nutritional assessment for patients with malignant tumors generally adopts anthropometric measurements, serum biochemical indicators, Nutritional Risk Screening 2002 (NRS2002), and Patient-Generated Subjective Global Assessment (PG-SGA). According to the expert consensus of the European Society of Parenteral and Enteral Nutrition (ESPEN), once patients are diagnosed with malignant tumors, NRS2002 is the first choice for screening and assessment of nutritional status, and PG-SGA is the preferred tool for assessment $(16,17)$. In addition, PG-SGA was also accepted as a nutritional assessment standard for cancer patients by the Oncology Nutritional Dietetic Practice Group of the American Diet Association (18).

However, there is no standard nutritional assessment method for newly diagnosed GIST patients. And there is no conclusion the preferred choice of assessment tool for nutritional status in GIST patients. Moreover, studies on the nutritional assessment of GIST patients are scarce, with only very few studies on the application of NRS2002 combined with PG-SGA in the evaluation of newly diagnosed GIST patients. Therefore, the purpose of this study is to use the NRS2002 combined with PSSGA score to evaluate the nutritional status of newly diagnosed
GIST patients and analyze the impact of their nutritional status on prognosis.

\section{METHODS AND MATERIALS}

\section{Patient Section}

This study retrospectively analyzed the medical data of 1,268 patients with newly diagnosed GIST admitted to the Fourth Hospital of Hebei Medical University from January 2014 to January 2018. Inclusion criteria were as follows: (1) pathological diagnosis was GIST; (2) without preoperative antitumor treatment; and (3) complete follow-up and clinical data. Exclusion criteria were as follows: (1) the patient had accepted antitumor therapies before surgery; (2) patients with cognitive impairment or other acute psychological problems; (3) without complete medical records and laboratory results; (4) the patient lost post-operative follow-up. This study was tested and approved by the ethics committee of The Fourth Hospital of Hebei Medical University, and the patients provided informed consent.

\section{Assessment Method}

All patients were screened by NRS2002 score after admission. The score $\geq 3$ indicates a risk of malnutrition in patients, which needs further assessment by PG-SGA. PG-SGA score includes patient self-assessment and medical staff assessment, which includes seven areas. Patients' self-assessment includes weight changes, dietary intake, self-reported symptoms, activities and function, and medical staff assessment includes nutritionrelated disease status, metabolic status, physical examination. Each of these seven areas is given a score of $0-4$, and the sum of scores obtained in each area is divided into quantitative and qualitative evaluations, thus providing guidance on the level of nutrition and drug intervention required by each patient. Quantitative evaluation is defined as follows: PG-SGA score of $0-1$ indicates that nutritional support not required and treatment in the future based on routine re-evaluation, 23 points indicate malnutrition or suspected malnutrition, 4-8 points indicate moderate malnutrition, and $\geq 9$ points indicate severe malnutrition. Qualitative evaluation indicates that patients with score of 4-8 need nutritional intervention and symptomatic treatment, and patients with score $\geq 9$ need urgent symptomatic treatment and appropriate nutritional support before antitumor treatment.

\section{Clinicopathological Parameters and Definitions}

We collected the basic data of newly diagnosed GIST patients including gender, age, weight, etc. Laboratory tests include routine blood tests and biochemical tests. Preoperative examination included abdominal computed tomography (CT), nuclear magnetic resonance imaging (MRI) and gastrointestinal endoscopy. Pathology and gene detection included tumor location, tumor size, mitotic count, immunohistochemistry, risk classification, c-kit exons 9, 11, 13 and 17, and PDGFRA exons 12 and 18 . The risk classification standard we adopted is the 2008 
version of the improved National Institutes of Health (NIH) classification (19).

\section{Follow-Up}

All patients were recommended to have a follow-up visit every 3 months in the first 2 years, and every 6 months after 2 years. All patients were followed up as outpatients. The latest follow-up date was in December 2020, and the median follow-up time was 68.6 months (range 16-76 months). The total survival time was calculated from diagnosis to death or the last followup. Overall survival (OS) was defined as time interval from operation to tumor-related death or last contact, and OS was the preferred destination.

\section{Statistical Analyses}

We provided PG-SGA standard questionnaires for all newly diagnosed GIST patients admitted to the Third Department of the Fourth Hospital of Hebei Medical University. Software of SPSS version 21.0 and GraphPad Prism 5.01 were utilized to perform statistical analyses. Anthropometric measurement and PG-SGA scores were expressed in the form of descriptive statistics (mean, standard deviation, and frequency), respectively. The $t$ test, ANOVA test, and correlation analysis were used to statistically evaluate the degree of correlation between these factors and the PG-SGA score. Survival analysis was performed using the Kaplan-Meier method. Univariate and multivariate analyses were investigated by the Cox proportional hazards regression model. The hazard ratio (HR) and 95\% confidence interval (CI) were used to assess relative risks. $P$ value $<0.05$ was regarded as statistical difference significantly.

\section{RESULTS}

\section{Clinicopathological Features of Newly Diagnosed GIST Patients}

A total of 1,268 patients were admitted from January 2014 to January 2018, including 887 cases (69.95\%) of tumors located in the stomach, 54 cases $(4.26 \%)$ in the duodenum, 235 cases (18.53\%) in the intestine, 30 cases $(2.37 \%)$ in the colon, and 62 cases $(4.89 \%)$ in the mesentery. All GIST patients were confirmed by pathology. There were 665 males (52.44\%) and 603 females $(47.56 \%)$, with a median age of $59(19-76)$ years old. The median diameter of the tumor was $6(2.3-15.4) \mathrm{cm}$, and the median nuclear mitotic figure was 5 (3-13) / 50HPF (Table 1).

\section{Nutritional Status of Newly Diagnosed GIST Patients}

Figure 1 presents the nutritional risk and assessment of 1,268 newly diagnosed GIST patients. A total of 1,268 newly diagnosed GIST patients were screened by NRS2002, and 986 patients $(77.76 \%)$ had the risk of malnutrition (NRS2002 score $\geq 3$ ), while 282 patients $(22.24 \%$ ) did not have the risk of malnutrition (NRS2002 score < 3). The PG-SGA evaluation of 1,268 patients showed that $64.11 \%$ of the patients scored $0-1$ points, indicating a good nutritional status, and nutritional support was not needed. $23.50 \%$ of the patients scored $2-3$ points, so only nutritional guidance was needed. And $10.09 \%$ of the patients scored $4-8$
TABLE 1 | General and tumor characteristics of study participants $(n=1268)$.

\begin{tabular}{|c|c|}
\hline Variables & $N$ (Percentage) \\
\hline Age (years) & $59.9 \pm 4.2^{*}$ \\
\hline Sex (male) & $665(52.44 \%)$ \\
\hline \multicolumn{2}{|l|}{ Weight loss } \\
\hline No WL (0-1.9\% of body weight) & $801(63.17 \%)$ \\
\hline Mild WL (2-2.9\% in 1-month or $2-5.9 \%$ in 6 months) & $208(16.40 \%)$ \\
\hline Moderate WL (3-4.9\% in 1-month or $6-9.9 \%$ in 6 months) & $117(9.23 \%)$ \\
\hline Severe WL (5-9.9\% in 1-month or10-19.9\% in 6 months) & $88(6.94 \%)$ \\
\hline Very severe WL (>10\% in 1-month or > 20\% in 6 months) & $54(4.26 \%)$ \\
\hline \multicolumn{2}{|l|}{ Tumor location } \\
\hline Stomach & $887(69.95 \%)$ \\
\hline Duodenum & $54(4.26 \%)$ \\
\hline Intestine & $235(18.53 \%)$ \\
\hline Colon & $30(2.37 \%)$ \\
\hline Mesentery & $62(4.89 \%)$ \\
\hline \multicolumn{2}{|l|}{ Tumor size (cm) } \\
\hline$<5.0$ & $383(30.21 \%)$ \\
\hline $5.0 \sim 10.0$ & $789(62.22 \%)$ \\
\hline$>10.0$ & 96 (7.57\%) \\
\hline \multicolumn{2}{|l|}{ Nuclear mitotic figure (50HPF) } \\
\hline$<5$ & 372 (29.34\%) \\
\hline $6 \sim 10$ & 708 (55.84\%) \\
\hline$>10$ & $188(14.83 \%)$ \\
\hline \multicolumn{2}{|l|}{ NIH classification } \\
\hline High risk & 279 (22.00\%) \\
\hline Moderate risk & $543(42.82 \%)$ \\
\hline Low risk & $309(24.37 \%)$ \\
\hline Very low risk & 137 (10.80\%) \\
\hline \multicolumn{2}{|l|}{ Ki-67 percentage } \\
\hline$\leq 10 \%$ & 848 (66.88\%) \\
\hline$>10 \%$ & $420(33.12 \%)$ \\
\hline \multicolumn{2}{|l|}{ c-kit exons } \\
\hline Positive & 961 (75.79\%) \\
\hline Negative & 307 (24.21\%) \\
\hline \multicolumn{2}{|l|}{ PDGFRA exons } \\
\hline Positive & $319(25.16 \%)$ \\
\hline Negative & 949 (74.84\%) \\
\hline
\end{tabular}

${ }^{*}$ Mean $\pm S D$.

points, indicating that there was mild/moderate malnutrition, and nutritional intervention and treatment were needed. 2.29\% patients scored $>9$ points, indicating severe malnutrition and urgent need for symptomatic treatment and adequate nutritional support. This study also found that only 117 (74.52\%) of the 157 patients who needed nutritional intervention (PG-SGA score $\geq$ 4) received nutritional support one week before the treatment. $2.37 \%$ of patients received parenteral nutrition (PN) support, $12.07 \%$ received enteral nutrition (EN) support, and $2.13 \%$ received both $\mathrm{EN}$ and $\mathrm{PN}$ support. In addition, we also found that 93 patients $(7.33 \%)$ with good nutrition (PG-SGA score < 4) received nutritional support treatment (Table 2 ).

According to 2008 version NIH stromal tumor risk classification standard, 1,268 patients were divided into 


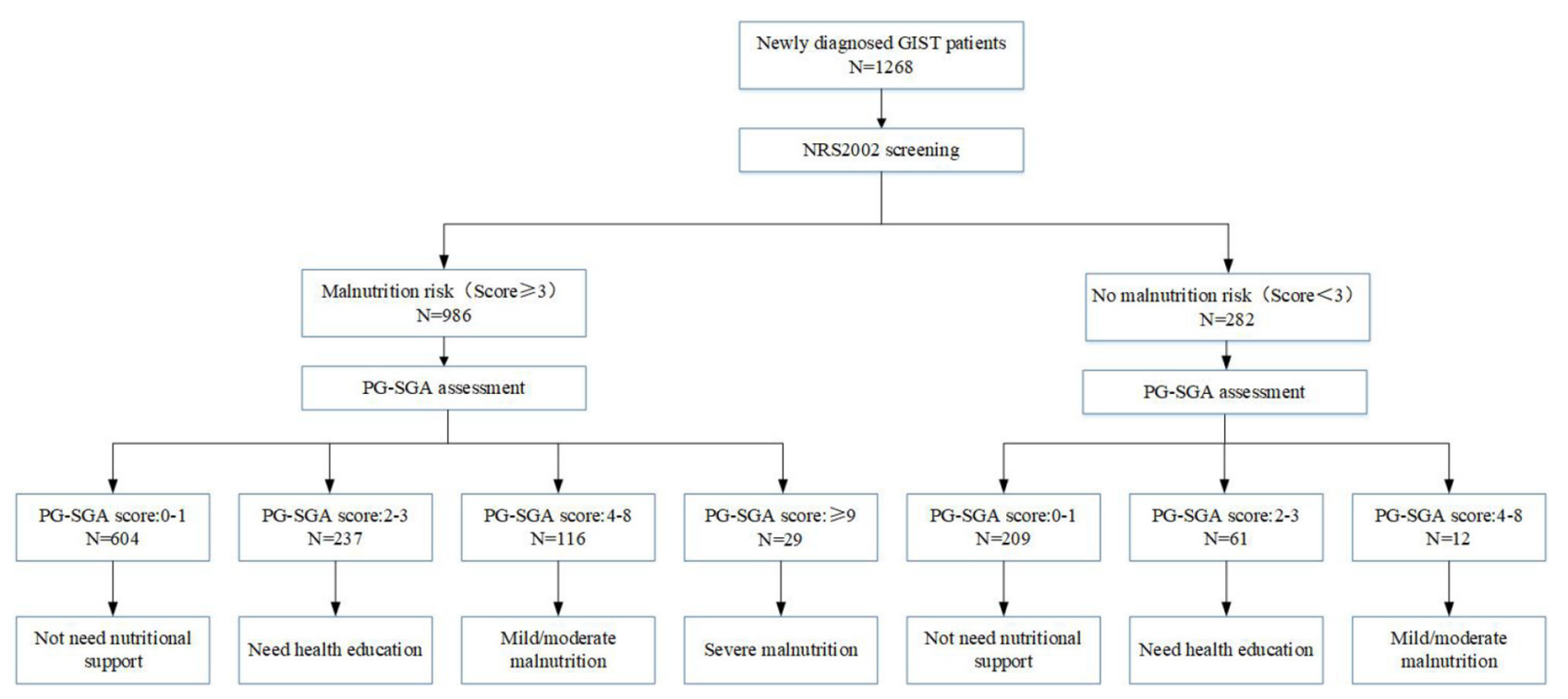

FIGURE 1 | Nutrition screening and assessment of 1,268 newly diagnosed GIST patients.

TABLE 2 | Patient-generated subjective global assessment classification and nutritional therapy situation $(N=1,268)$.

\begin{tabular}{|c|c|c|c|c|c|}
\hline \multirow[t]{2}{*}{ Nutrition support } & \multirow[t]{2}{*}{ Total (\%) } & \multicolumn{4}{|c|}{ PG-SGA } \\
\hline & & $0 \sim 1(\%)$ & $2 \sim 3(\%)$ & $4 \sim 8(\%)$ & $\geqq 9(\%)$ \\
\hline No & $1058(83.43)$ & 779 (95.82) & $239(80.20)$ & 38 (29.69) & $2(6.90)$ \\
\hline Yes & & & & & \\
\hline PN & 30 (2.37) & $0(0)$ & $5(1.68)$ & 14 (10.94) & 11 (37.93) \\
\hline EN & $153(12.07)$ & $34(4.18)$ & $52(17.45)$ & $57(44.53)$ & $10(34.48)$ \\
\hline
\end{tabular}

PN, parenteral nutrition; EN, enteral nutrition; PG-SGA, patient-generated subjective global assessment.

TABLE 3 | The relationship between risk classification and incidence of nutritional risk in newly diagnosed GIST patients $(N=1,268)[n(\%)]$.

\begin{tabular}{|c|c|c|c|c|c|c|}
\hline \multirow[t]{2}{*}{ Group } & \multirow[t]{2}{*}{$N$} & \multicolumn{4}{|c|}{ PG-SGA } & \multirow[t]{2}{*}{ Malnutrition incidence } \\
\hline & & $0 \sim 1(\%)$ & $2 \sim 3(\%)$ & $4 \sim 8(\%)$ & $\geq 9(\%)$ & \\
\hline High risk & 279 & 91 (32.62) & 99 (35.48) & $65(12.54)$ & $24(8.60)$ & $188(67.38)$ \\
\hline Low risk & 309 & $269(87.06)$ & $33(10.68)$ & $7(2.27)$ & $0(0)$ & $40(12.94)^{\star}$ \\
\hline Very low risk & 137 & 130 (94.89) & $7(5.11)$ & $0(0)$ & $0(0)$ & $7(5.11)^{\star}$ \\
\hline
\end{tabular}

*Compared with high risk group, two-sided chi-square test, all $p<0.05$.

TABLE 4 | Location of gastrointestinal stromal tumors and incidence of nutritional risk ( $N=1,268)[n(\%)]$.

\begin{tabular}{|c|c|c|c|c|c|c|}
\hline \multirow[t]{2}{*}{ Group } & \multirow[t]{2}{*}{$N$} & \multicolumn{4}{|c|}{ PG-SGA } & \multirow[t]{2}{*}{ Malnutrition incidence } \\
\hline & & $0 \sim 1(\%)$ & $2 \sim 3(\%)$ & $4 \sim 8(\%)$ & $\geq 9(\%)$ & \\
\hline Stomach & 887 & $605(68.21)$ & $180(20.29)$ & 88 (9.92) & $14(1.58)$ & $282(46.61)^{\star}$ \\
\hline Duodenum & 54 & $29(53.70)$ & $19(35.19)$ & $5(9.26)$ & $1(1.85)$ & $25(46.30)^{\star}$ \\
\hline Intestine & 235 & $139(59.15)$ & $73(31.06)$ & $17(7.23)$ & $6(2.55)$ & $96(40.85)^{\star}$ \\
\hline Colon & 30 & $20(66.67)$ & $6(20.00)$ & $3(10.00)$ & $1(3.33)$ & $10(33.33)^{\star}$ \\
\hline
\end{tabular}

${ }^{*}$ Compared with mesentery group, two-sided chi-square test, all $p<0.05$. 
four groups. There were 137 patients in the extremely low risk group, $7(5.11 \%)$ of whom were at risk of malnutrition. There were 309 cases in the low-risk group, of which 40 (12.94\%) patients had the risk of malnutrition. There were 543 patients in the moderate-risk group, 220 (40.52\%) of whom were at risk of malnutrition. There were 279 cases in the high-risk group, of which $188(67.38 \%)$ patients had the risk of malnutrition. The comparison between groups showed that the risk of malnutrition in the high-risk group was significantly higher than that in the other three groups $(p<0.05)$ (Table 3 ).

A total of 1,268 patients were divided into five groups according to the location of tumor. Among the 887 cases of gastric stromal tumors, 282 cases $(46.61 \%)$ had malnutrition risk, while 96 of the 235 patients with intestinal stromal tumors were at risk of malnutrition $(40.85 \%)$. There were 54 patients with duodenal tumors and 30 patients with colonic tumors, with 25 cases $(46.30 \%)$ and 10 cases $(33.33 \%)$ at risk of malnutrition, respectively. Meanwhile, there were 62 cases of stromal tumors in mesentery, and 42 cases (67.74\%) had malnutrition risk. The comparison among groups showed that the risk of malnutrition in patients with mesentery stromal tumors was significantly higher $(p<0.05)$ (Table 4$)$.

\section{Analysis of Related Factors Affecting PG-SGA Score}

Our study revealed the relationship between PG-SGA of newly diagnosed GIST patients scores and possible related factors. The PG-SGA related factors were patients' age $(p=0.013)$, BMI $(p$ $=0.001)$, weight loss $(p=0.001)$, anemia $(p=0.005)$, prealbumin $(p=0.010)$, albumin $(p=0.002)$, tumor location $(p=$ $0.001)$, tumor size $(p=0.002)$, and NIH classification $(p=0.001)$ (Table 5).

\section{Treatment and Prognosis of Newly Diagnosed GIST Patients}

Among 1,268 newly diagnosed GIST patients, 1,046 patients $(82.49 \%)$ underwent direct surgical resection, and 222 patients (17.50\%) received imatinib targeted therapy due to large tumor size. All patients were followed up for a median of 68.6 months (range 16-86). The 5-year OS rate was $74.61 \%$, and the median survival time was 42.7 months (range 13-74). The 5-year OS rate of patients with NRS2002 score $<3$ was $79.79 \%$, while that of patients with NRS2002 score $\geq 3$ was only $73.12 \%(p$ $=0.007$, Figure 2A). According to different PG-SGA scores, the stratified analysis of 1,268 patients with PG-SGA evaluation showed that the 5-year OS of patients with PG-SGA score of $0-1$ and $2-3$ was 78.60 and $73.93 \%$, respectively, which was significantly better than that of patients with PG-SGA score $>3(p<0.001$, Figure 2B). The prognosis of GIST patients with different PG-SGA scores in nutritional therapy and without any intervention was shown in Figure 3. For patients with PG-SGA score $<4$, there was no significant difference in prognosis between nutritional therapy and nonnutritional intervention $(p=0.164,0.251)$. However, for patients with PG-SGA score of $4 \sim 8$, especially those with PGSGA score $\geq 9$, nutritional therapy significantly improved
TABLE 5 | Analysis of PG-SGA score with factors affecting nutritional status.

\begin{tabular}{|c|c|c|c|c|}
\hline Characteristic & Cases (n) & $\begin{array}{l}\text { PG-SGA } \\
\text { score } \\
\text { (Median } \\
\pm \text { SD) }\end{array}$ & $\begin{array}{l}\text { Statistical } \\
\text { value }\end{array}$ & $P$-value \\
\hline Age (years) & & & $u=-4.041$ & 0.013 \\
\hline$\geq 60$ & 718 & $5 \pm 1.66$ & & \\
\hline$<60$ & 550 & $3 \pm 1.32$ & & \\
\hline Sex & & & $t=1.549$ & 0.083 \\
\hline Male & 665 & $4 \pm 1.47$ & & \\
\hline Female & 603 & $3 \pm 1.31$ & & \\
\hline $\mathrm{BMI}\left(\mathrm{kg} / \mathrm{m}^{2}\right)$ & & & $u=11.421$ & 0.001 \\
\hline$<18.5$ & 155 & $6 \pm 2.19$ & & \\
\hline $18.5 \sim 25.0$ & 878 & $4 \pm 1.11$ & & \\
\hline$>25.0$ & 235 & $3 \pm 0.86$ & & \\
\hline Weight loss & & & $u=14.671$ & 0.001 \\
\hline $\begin{array}{l}\text { No WL (<2\% of body } \\
\text { weight) }\end{array}$ & 801 & $3 \pm 0.87$ & & \\
\hline $\begin{array}{l}\text { Mild WL (2-3\% in } 1 \text {-month } \\
\text { or } 2-6 \% \text { in } 6 \text { months) }\end{array}$ & 208 & $4 \pm 1.89$ & & \\
\hline $\begin{array}{l}\text { Moderate WL }(3-5 \% \text { in } \\
1 \text {-month or } 6-10 \% \text { in } 6 \\
\text { months) }\end{array}$ & 117 & $5 \pm 1.36$ & & \\
\hline $\begin{array}{l}\text { Severe WL }(5-10 \% \text { in } \\
1 \text {-month or10-20\% in } 6 \\
\text { months) }\end{array}$ & 88 & $6 \pm 2.07$ & & \\
\hline $\begin{array}{l}\text { Very severe } \mathrm{WL}(>10 \% \text { in } \\
1 \text {-month or }>20 \% \text { in } 6 \\
\text { months) }\end{array}$ & 54 & $8 \pm 1.25$ & & \\
\hline Anemia & & & $t=9.997$ & 0.005 \\
\hline Yes & 494 & $5 \pm 1.76$ & & \\
\hline No & 774 & $4 \pm 0.88$ & & \\
\hline Pre-albumin (mg/dL) & & & $t=23.043$ & 0.010 \\
\hline$<20$ & 390 & $6 \pm 1.34$ & & \\
\hline$\geq 20$ & 878 & $4 \pm 1.11$ & & \\
\hline Albumin(g/L) & & & $t=11.034$ & 0.002 \\
\hline$<35$ & 338 & $5 \pm 1.76$ & & \\
\hline$\geq 35$ & 930 & $3 \pm 0.97$ & & \\
\hline Tumor location & & & $u=10.876$ & 0.001 \\
\hline stomach & 887 & $3 \pm 1.71$ & & \\
\hline duodenum & 54 & $4 \pm 1.38$ & & \\
\hline intestine & 235 & $5 \pm 1.65$ & & \\
\hline colon & 30 & $5 \pm 1.13$ & & \\
\hline mesentery & 62 & $7 \pm 1.44$ & & \\
\hline Tumor size (cm) & & & $u=13.573$ & 0.002 \\
\hline$<5.0$ & 383 & $2 \pm 1.02$ & & \\
\hline $5.0 \sim 10.0$ & 789 & $4 \pm 0.96$ & & \\
\hline$>10.0$ & 96 & $7 \pm 2.15$ & & \\
\hline $\mathrm{NIH}$ classification & & & $u=15.621$ & 0.001 \\
\hline High risk & 279 & $7 \pm 2.08$ & & \\
\hline Middle risk & 543 & $5 \pm 1.17$ & & \\
\hline Low risk & 309 & $3 \pm 0.88$ & & \\
\hline Very low risk & 137 & $3 \pm 1.22$ & & \\
\hline
\end{tabular}

the prognosis of patients, and the survival time was better than that of patients without nutritional intervention $(p=$ $0.025,0.001)$. 

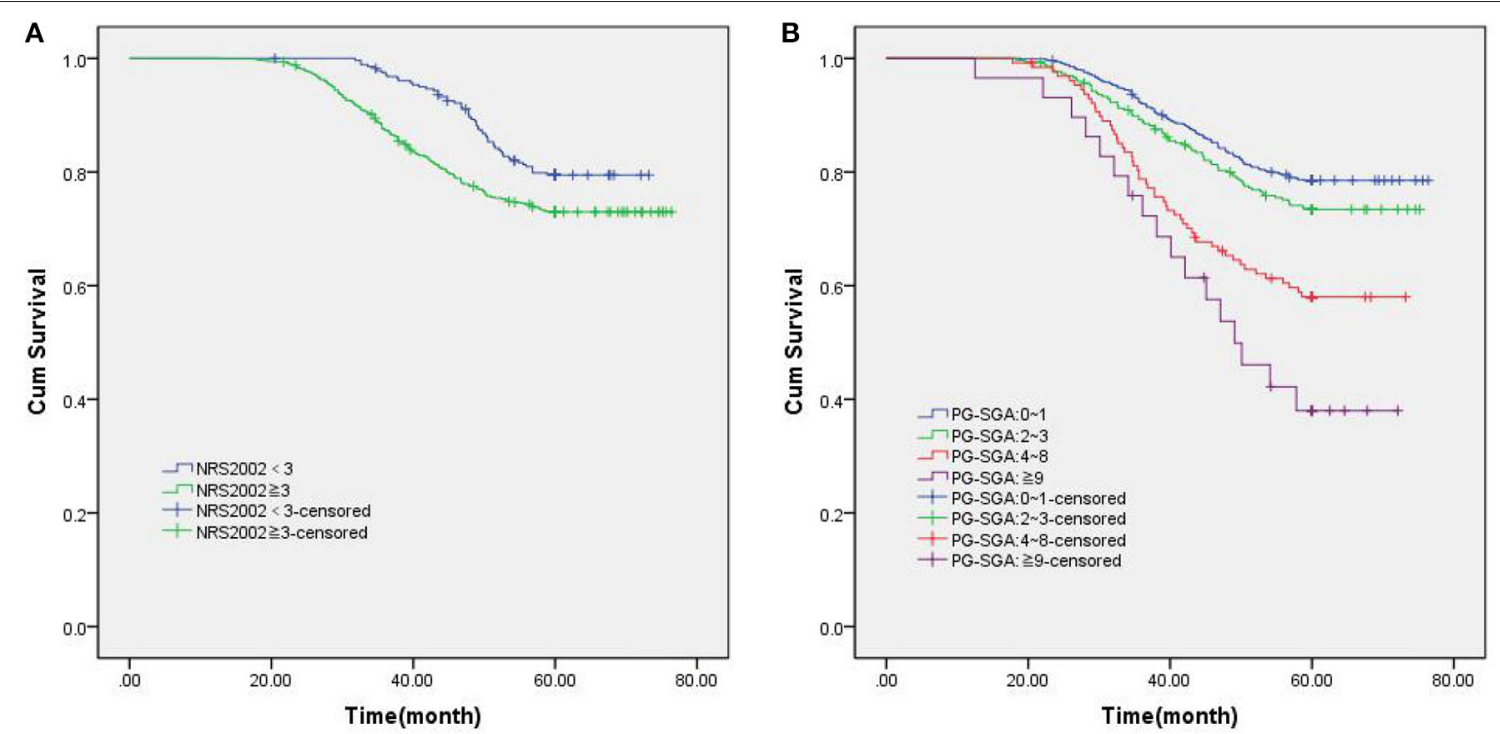

FIGURE 2 | Kaplan-Meier survival curves in patients with newly diagnosed GIST patients. (A) Overall survival based on NRS2002 scores; (B) Overall survival based on PG-SGA scores.

Univariate and multivariate analysis showed that PG-SGA score $(p=0.001, \mathrm{HR}=1.638,95 \% \mathrm{CI}$ : $1.259-2.441)$, pre-albumin $(p=0.033, \mathrm{HR}=0.687,95 \% \mathrm{CI}: 0.548-0.861), \operatorname{BMI}(p=0.011$, $\mathrm{HR}=1.321,95 \% \mathrm{CI}: 0.925-1.874), \mathrm{NIH}$ classification $(p=0.000$, $\mathrm{HR}=2.805,95 \% \mathrm{CI}: 2.241-3.510)$, nutritional therapy $(p=0.012$, $\mathrm{HR}=1.267$, 95\%CI: 0.987-1.762) were independent risk factors affecting the 5-year OS rate of newly diagnosed GIST patients (Table 6).

\section{DISCUSSION}

GIST is the most common mesenchymal tumor in the gastrointestinal tract, accounting for about $2 \%$ of gastrointestinal tumors, which can occur throughout the gastrointestinal tract, also can occur in the mesangium, pelvis and retroperitoneum (20, 21). Due to the lack of specific manifestations of GIST, clinical symptoms such as gastrointestinal bleeding, abdominal pain and discomfort or abdominal mass often occur (22). The best time for early treatment has been delayed after symptom deterioration, and the 5-year survival rate has been greatly affected. Meanwhile, cancer patients are often accompanied by malnutrition in the initial diagnosis, especially digestive tract malignant tumors (23).

Our retrospective study was the first to investigate the nutritional status of newly diagnosed GIST patients and possible factors leading to malnutrition by NRS2002 combined with PG-SGA score. We found that $12.38 \%$ of newly diagnosed GIST patients were malnourished, and $2.29 \%$ of them needed urgent management to relieve malnutritional symptom and/or nutritional support. This is similar to the results of previous study conducted by Guo et al. (24) that the incidence of malnutrition was $12 \%$, and the risk of malnutrition was $34 \%$. Our results also discovered that malnutrition was common in newly diagnosed GIST patients, and these patients would need prompt nutrition and dietician education and guidance. This may be due to recurrent gastrointestinal symptoms such as anorexia and anorexia in GIST patients, which further leads to weight loss. In addition, the rapid growth of tumors, abdominal recurrence and systemic metastasis increased nutrition consumption, resulting in malnutrition in patients. Moreover, we found that the risk of malnutrition in patients in the high-risk group and in patients with tumors located in the mesentery was significantly higher than that in other groups.

We further analyzed the factors affecting PG-SGA score and found that tumor location and tumor risk classification were independent risk factors. Furthermore, we found that the PGSGA score was closely related to the OS of patients. The 5-year OS rates of patients with $0-1$ score were better than those of other groups, especially those with $\geq 4$ scores. The higher PGSGA score was associated with worse prognosis. These findings have also been supported by other studies. Ge et al. found that the nutritional status of patients with gastrointestinal cancer determines the quality of life during subsequent treatment (18). In addition, Tan et al. found that nutritional status assessed by PG-SGA may be a predictor of prognosis in patients with advanced cancer, especially in patients with gastrointestinal tumors (25). In view of these results, we speculate that PG-SGA score may exert a more effective value in evaluating the prognosis of newly diagnosed GIST patients.

There are still some limitations in our research. First, this study was a single-center retrospective study with limited number of cases. Second, only OS was investigated due to the lack of data on progression-free survival and quality of life. Therefore, further multicenter, prospective studies to more comprehensively assess the prediction values of NRS2002 combined with PG-SGA score on risk of adverse clinical outcomes in patients with GIST are still needed. 

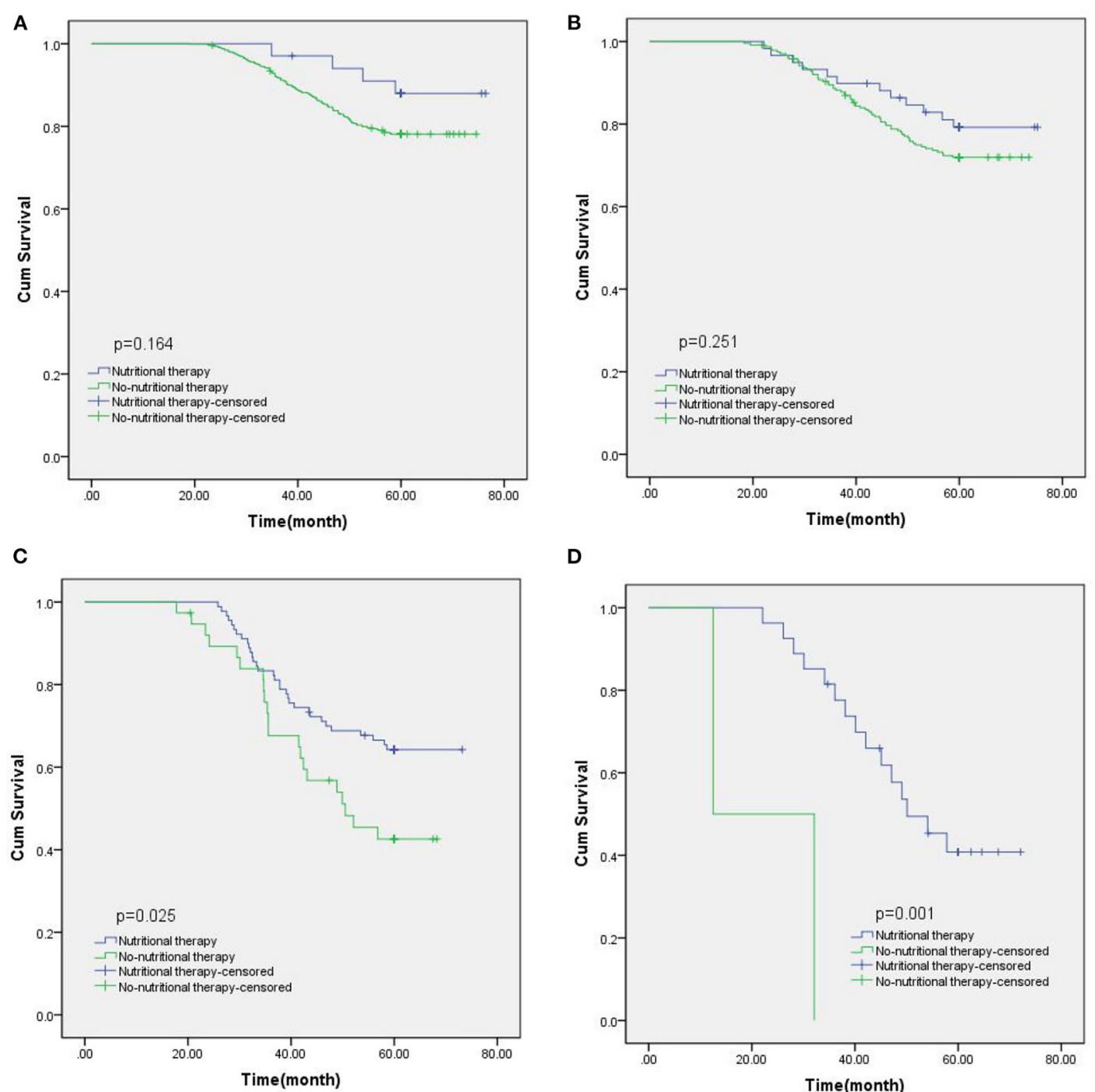

D

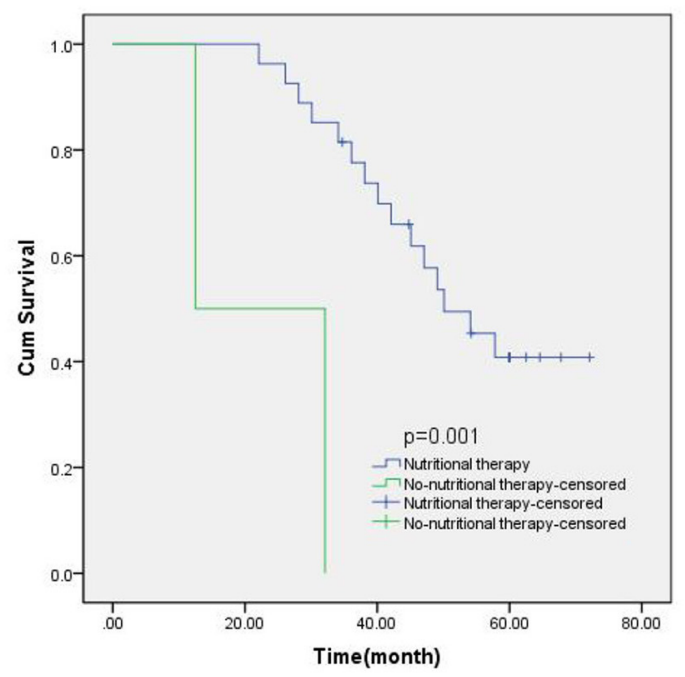

FIGURE 3 | The prognosis of GIST patients with different PG-SGA scores in nutritional therapy and without any intervention. (A) PG-SGA scores: 0 1; (B) PG-SGA scores: 2 3; (C) PG-SGA scores: 4 8; (D) PG-SGA scores: $\geq 9$.

TABLE 6 | Univariate and multivariate analyses for OS in patients with newly diagnosed GIST.

\section{Variables}

Gender (female vs. male)

Age ( $\leq 60$ vs. $>60)$

NRS2002(<3 vs. $\geq 3$ )

PG-SGA (0-1 vs. $2-3$ vs. $4-8$ vs. $\geq 9$ )

Anemia (Yes vs. no)

Pre-albumin (mg/dL) (<20 vs. $\geq 20)$

Albumin (g/L) (<35 vs. $\geq 35)$

$\mathrm{BMI}(<18.5$ vs. $\geq 18.5$ and $<25.0$ vs. $\geq 25.0$ )

$\mathrm{NIH}$ classification (High risk vs. moderate risk vs. low risk vs. very low risk)

Nutritional therapy (No vs. yes)
Univariate analysis

\begin{tabular}{|c|c|c|c|c|c|}
\hline$P$ value & HR & $95 \% \mathrm{Cl}$ & $P$ value & HR & $95 \% \mathrm{Cl}$ \\
\hline 0.010 & 1.321 & $0.898-1.624$ & 0.079 & 0.758 & $0.556-1.003$ \\
\hline 0.065 & 1.457 & $1.123-2.083$ & - & - & - \\
\hline 0.033 & 1.593 & $1.256-2.763$ & 0.066 & 0.895 & $0.431-1.346$ \\
\hline 0.008 & 1.832 & 1.360-3.321 & 0.001 & 1.638 & $1.259-2.441$ \\
\hline 0.077 & 0.653 & $0.489-1.032$ & - & - & - \\
\hline 0.048 & 1.032 & $0.783-1.439$ & 0.033 & 0.687 & $0.548-0.861$ \\
\hline 0.031 & 1.329 & $1.102-1.876$ & 0.054 & 0.772 & 0.542-1.302 \\
\hline 0.022 & 1.487 & $1.212-2.034$ & 0.011 & 1.321 & $0.925-1.874$ \\
\hline 0.001 & 3.458 & 2.198-5.329 & 0.000 & 2.805 & $2.241-3.510$ \\
\hline 0.032 & 1.432 & $1.031-1.987$ & 0.012 & 1.267 & $0.987-1.762$ \\
\hline
\end{tabular}




\section{CONCLUSION}

In this study, NRS2002 combined with PG-SGA score was used to evaluate the nutritional status of newly diagnosed GIST patients in China for the first time to the best of our knowledge. About $12.38 \%$ of GIST patients had malnutrition at the time of diagnosis, and more than $1 / 10$ of GIST patients needed urgent nutritional intervention and management. More attention should be paid to the nutritional status of GIST patients, especially those with high risk of malnutrition, such as elderly patients and tumors located in the mesenteric. These highrisk patients should be timely PG-SGA assessment, and give nutritional education and necessary nutritional support.

\section{DATA AVAILABILITY STATEMENT}

The raw data supporting the conclusions of this article will be made available by the authors, without undue reservation.

\section{ETHICS STATEMENT}

This study was tested and approved by the Ethics Committee of The Fourth Hospital of Hebei Medical University, and the

\section{REFERENCES}

1. Cioffi A, Maki RG. GI stromal tumors: 15 years of lessons from a rare cancer. J Clin Oncol. (2015) 33:1849-54. doi: 10.1200/JCO.2014.59.7344

2. von Mehren M, Joensuu H. Gastrointestinal stromal tumors. J Clin Oncol. (2018) 36:136-43. doi: 10.1200/JCO.2017.74.9705

3. Corless CL, Barnett CM, Heinrich MC. Gastrointestinal stromal tumours: origin and molecular oncology. Nat Rev Cancer. (2011) 11:865-78. doi: 10.1038/nrc3143

4. Abe Vicente M, Barão K, Silva TD, Forones NM. What are the most effective methods for assessment of nutritional status in outpatients with gastric and colorectal cancer? Nutr Hosp. (2013) 28:585-91. doi: 10.1093/annonc/mdt203.228

5. Malihi Z, Kandiah M, Chan YM, Hosseinzadeh M, Sohanaki Azad M, Zarif Yeganeh M. Nutritional status and quality of life in patients with acute leukaemia prior to and after induction chemotherapy in three hospitals in Tehran, Iran: a prospective study. J Hum Nutr Diet. (2013) 26:12331. doi: $10.1111 /$ jhn. 12043

6. Wie GA, Cho YA, Kim SY, Kim SM, Bae JM, Joung H. Prevalence and risk factors of malnutrition among cancer patients according to tumor location and stage in the National Cancer Center in Korea. Nutrition. (2010) 26:2638. doi: 10.1016/j.nut.2009.04.013

7. Lis CG, Gupta D, Lammersfeld CA, Markman M, Vashi PG. Role of nutritional status in predicting quality of life outcomes in cancera systematic review of the epidemiological literature. Nutr J. (2012) 11:27. doi: 10.1186/1475-2891-11-27

8. Kaikani W, Bachmann P. Consequences of a comorbidity often neglected in oncology: malnutrition. Bull Cancer. (2009) 96:659-64. (French). doi: 10.1684/bdc.2009.0875

9. Laky B, Janda M, Kondalsamy-Chennakesavan S, Cleghorn G, Obermair A. Pretreatment malnutrition and quality of life - association with prolonged length of hospital stay among patients with gynecological cancer: a cohort study. BMC Cancer. (2010) 10:232. doi: 10.1186/1471-2407-10-232

10. Datema FR, Ferrier MB, Baatenburg de. Jong RJ. Impact of severe malnutrition on short-term mortality and overall survival in head and neck cancer. Oral Oncol. (2011) 47:910-4. doi: 10.1016/j.oraloncology.2011. 06.510 patients provided informed consent. The patients/participants provided their written informed consent to participate in this study.

\section{AUTHOR CONTRIBUTIONS}

QZ: conception and design, and administrative support. PD, PY, YT, HG, and YLi: provision of study materials or patients. PD, PY, YT, and HG: collection and assembly of data. PD and CS: data analysis and interpretation. PD, HG, PY, CS, YT, YLiu, YLi, and QZ: manuscript writing. All authors contributed to the article and approved the submitted version.

\section{FUNDING}

This work was supported by the Cultivating Outstanding Talents Project of Hebei Provincial Government Fund (No. 2019012); Hebei public health committee county-level public hospitals suitable health technology promotion and storage project (No. 2019024); Hebei Medical University Education and Teaching Research Project (Nos. 2020CGPY-12, 2020CHYB23); Hebei University Science and Technology Research Project (No. ZD2019139).

11. Van Cutsem E, Arends J. The causes and consequences of cancerassociated malnutrition. Eur J Oncol Nurs. (2005) 9(Suppl. 2):S5163. doi: 10.1016/j.ejon.2005.09.007

12. Gabrielson DK, Scaffidi D, Leung E, Stoyanoff L, Robinson J, Nisenbaum R, et al. Use of an abridged scored Patient-Generated Subjective Global Assessment (abPG-SGA) as a nutritional screening tool for cancer patients in an outpatient setting. Nutr Cancer. (2013) 65:234-9. doi: 10.1080/01635581.2013.755554

13. Gupta D, Vashi PG, Lammersfeld CA, Braun DP. Role of nutritional status in predicting the length of stay in cancer: a systematic review of the epidemiological literature. Ann Nutr Metab. (2011) 59:96-106. doi: 10.1159/000332914

14. Ho MY, Blanke CD. Gastrointestinal stromal tumors: disease and treatment update. Gastroenterology. (2011) 140:13726.e2. doi: 10.1053/j.gastro.2011.03.017

15. Valsangkar N, Sehdev A, Misra S, Zimmers TA, O’Neil BH, Koniaris LG. Current management of gastrointestinal stromal tumors: Surgery, current biomarkers, mutations, and therapy. Surgery. (2015) 158:114964. doi: 10.1016/j.surg.2015.06.027

16. Jager-Wittenaar H, Ottery FD. Assessing nutritional status in cancer: role of the patient-generated subjective global assessment. Curr Opin Clin Nutr Metab Care. (2017) 20:322-29. doi: 10.1097/MCO.0000000000000389

17. Bauer J, Capra S, Ferguson M. Use of the scored Patient-Generated Subjective Global Assessment (PG-SGA) as a nutrition assessment tool in patients with cancer. Eur J Clin Nutr. (2002) 56:779-85. doi: 10.1038/sj.ejcn.1601412

18. Ge T, Lin T, Yang J, Wang M. Nutritional status and related factors of patients with advanced lung cancer in northern China: a retrospective study. Cancer Manag Res. (2019) 11:2225-31. doi: 10.2147/CMAR.S193567

19. Joensuu H. Risk stratification of patients diagnosed with gastrointestinal stromal tumor. Hum Pathol. (2008) 39:14119. doi: 10.1016/j.humpath.2008.06.025

20. Machairas A, Karamitopoulou E, Tsapralis D, Karatzas T, Machairas N, Misiakos EP. Gastrointestinal stromal tumors (GISTs): an updated experience. Dig Dis Sci. (2010) 55:3315-27. doi: 10.1007/s10620-010-1360-9

21. Faigel DO, Abulhawa S. Gastrointestinal stromal tumors: the role of the gastroenterologist in diagnosis and risk stratification. J Clin Gastroenterol. (2012) 46:629-36. doi: 10.1097/MCG.0b013e3182548f6c 
22. Badalamenti G, Rodolico V, Fulfaro F, Cascio S, Cipolla C, Cicero G, et al. Gastrointestinal stromal tumors (GISTs): focus on histopathological diagnosis and biomolecular features. Ann Oncol. (2007) 18:vi136-40. doi: 10.1093/annonc/mdm243

23. Song C, Cao J, Zhang F, Wang C, Guo Z, Lin Y, et al. Nutritional risk assessment by scored patient-generated subjective global assessment associated with demographic characteristics in 23,904 common malignant tumors patients. Nutr Cancer. (2019) 71:50-60. doi: 10.1080/01635581.2019.1566478

24. Guo ZQ, Yu JM, Li W, Fu ZM, Lin Y, Shi YY, et al. Investigation on the Nutrition Status and Clinical Outcome of Common Cancers (INSCOC) group. Survey and analysis of the nutritional status in hospitalized patients with malignant gastric tumors and its influence on the quality of life. Support Care Cancer. (2020) 28:373-80. doi: 10.1007/s00520-019-04803-3

25. Tan CS, Read JA, Phan VH, Beale PJ, Peat JK, Clarke SJ. The relationship between nutritional status, inflammatory markers and survival in patients with advanced cancer: a prospective cohort study. Support Care Cancer. (2015) 23:385-91. doi: 10.1007/s00520-014-2385-y
Conflict of Interest: The authors declare that the research was conducted in the absence of any commercial or financial relationships that could be construed as a potential conflict of interest.

Publisher's Note: All claims expressed in this article are solely those of the authors and do not necessarily represent those of their affiliated organizations, or those of the publisher, the editors and the reviewers. Any product that may be evaluated in this article, or claim that may be made by its manufacturer, is not guaranteed or endorsed by the publisher.

Copyright (c) 2021 Ding, Guo, Yang, Sun, Tian, Liu, Li and Zhao. This is an openaccess article distributed under the terms of the Creative Commons Attribution License (CC BY). The use, distribution or reproduction in other forums is permitted, provided the original author(s) and the copyright owner(s) are credited and that the original publication in this journal is cited, in accordance with accepted academic practice. No use, distribution or reproduction is permitted which does not comply with these terms. 